\title{
The Last-Surviving Moroccan Dorcas Gazelle (Gazella dorcas massaesyla) Viability Analysis [M'Sabih Talaa Reserve, Morocco]
}

\author{
Ait Baamrane Moulay Abdeljalil1,4*, Znari Mohammed², El Mercht Said ${ }^{3}$, Bellout Siham ${ }^{4}$ and \\ Naimi Mohamed ${ }^{5}$ \\ ${ }_{1}^{1}$ Faculty of Applied Sciences Ait Melloul, Ibn Zohr University - Agadir, Morocco \\ ${ }^{2}$ Biodiversity and Ecosystem Dynamics, Faculty of Sciences-Semlalia, Cadi Ayyad University, Marrakech, Morocco \\ 3 Regional Center for Forestery Research, Marrakech, Morocco \\ ${ }^{4}$ Biodiversity and Ecosystems Functioning, Faculty of Sciences, Ibn Zohr University, Agadir, Morocco \\ 5 Polydisciplinar faculty, Sultan Moulay Slimane University, Beni Mellal, Morocco \\ *Corresponding Author
}

Received: 9th April, 2021

Accepted: 7th May, 2021

Published online: $12^{\text {th }}$ May, 2021

https://doi.org/10.33745/ijzi.2021.v07i01.017

\begin{abstract}
The single remnant Moroccan dorcas gazelle (Gazella dorcas massaesyla) has been isolated for more than five decades in the M'Sabih Talaa reserve, an arid area of west-central Morocco. This population has been subject to different disturbances, especially poaching and depredation by feral dogs. A five-season line transect survey revealed that the population size has been halved in less than 15 years with a lower apparent fecundity rate. In the present work, we carried out a population viability analysis simulating different scenarios using VORTEX software based on available demographic and life-history data from captive populations.. A sensitivity analysis revealed that inbreeding depression and possible catastrophic events could have a considerable impact on the population's prospects. Scenarios of splitting population into two subpopulations with different management measures reducing/deleting mortality sources, is proposed. Such scenarios resulted in reducing the consequences of catastrophic events would significantly mitigate the harmful effects of both inbreeding and environmental stochasticity. These results may be of a general interest to conservationists dealing with this unique and imperiled population.
\end{abstract}

Keywords: Wildlife management, Gazella dorcas massaesyla, Sensitive analysis, Vortex software, Inbreeding depression

Citation: Ait Baamrane Moulay Abdeljalil, Znari Mohammed, El Mercht Said, Bellout Siham and Naimi Mohamed: The last-surviving Moroccan dorcas gazelle (Gazella dorcas massaesyla) viability analysis [M'Sabih Talaa Reserve, Morocco]. Intern. J. Zool. Invest. 7 (1): 201-216, 2021.

https://doi.org/10.33745/ijzi.2021.v07i01.017 


\section{Introduction}

During the last century, North African ungulate species had suffered from overhunting and habitat loss due to overgrazing and agriculture intensification (Loggers et al., 1992; Cuzin, 2003). Among these species, the dorcas gazelle Gazella dorcas L. 1758 (Antilopinae subfamily) which is globally considered as vulnerable (VU) (IUCN 2008), has been classified as critically endangered in Morocco (Cuzin, 1996, 2003) and has declined precipitously throughout the country, due primarily to poaching, excessive hunting and severe habitat degradation as a consequence of the expansion of permanent agriculture and increased livestock grazing pressures. The Moroccan dorcas gazelle G. d. massaesyla (Cabrera, 1928), which is endemic to the Moroccan Plateaux's Atlantic coast north of the Atlas range (Panouse, 1957; Gentry, 1971; Alados, 1987), has persisted as the single remnant and isolated population within a 1987 ha reserve established since 1952 at M'Sabih Talaa (hereafter MT), west-central Morocco (Fig. 1). The reserve's perimeter has been fenced since 1960 to exclude poachers and livestock grazing. Currently, the gazelle population in MT is facing seasonal, albeit low-intensity livestock grazing and associated disturbance in the form of the occasional presence of livestock (sheep and goats) illegally introduced by the shepherds into the reserve throughout the damaged parts of the fence, continue to be important threats to the gazelle. Feral dogs and periodic poaching are still the major mortality factors on the reserve (Loggers, 1992; Ait Baamrane et al., 2009, 2013). Disease transmission from livestock remains a potential threat to the gazelle. The "Peste des petits ruminant" (PPR), which is a fatal disease specifically affecting small ruminants, has been reported in the livestock in Morocco. Captive dorcas gazelle was already the subject of contamination in African zoos and of a severe outbreak in Saudi Arabia (Housawi et al., 2004). Finally, effect of drought on the population may be significant.

The MT gazelle population continued to be relatively neglected from a conservation viewpoint during more than a half century. This remnant population might be subject to extinction unless a population recovery program and habitat management are initiated urgently. Indeed, conservation and management of such a small isolated population commonly face problems like inbreeding depression, genetic drift and high susceptibility to catastrophes, diseases and environmental stochasticity.

The population size in MT seems rather to fluctuate. In 1987, the population size estimate was about 209 animals (Loggers, 1992). Ten years later (in 1996), the population numbered 184 individuals (Marraha, 1996). The recent study conducted in 2009 using line transect sampling method gives a population size of less than 100 individuals (Ait Baamrane et al., 2013). Any bottleneck and a very low long time effective population size would cause inbreeding which is likely to further diminish the animals' fitness. Population genetic analyses based on mtDNA and microsatellites found a low genetic variability in the MT population comparatively to other dorcas gazelle semicaptive and captive populations (Lerp et al., 2011; Godinho et al., 2012).

In the present study, we carried out a population viability analysis based on available demographic, biological and genetic 


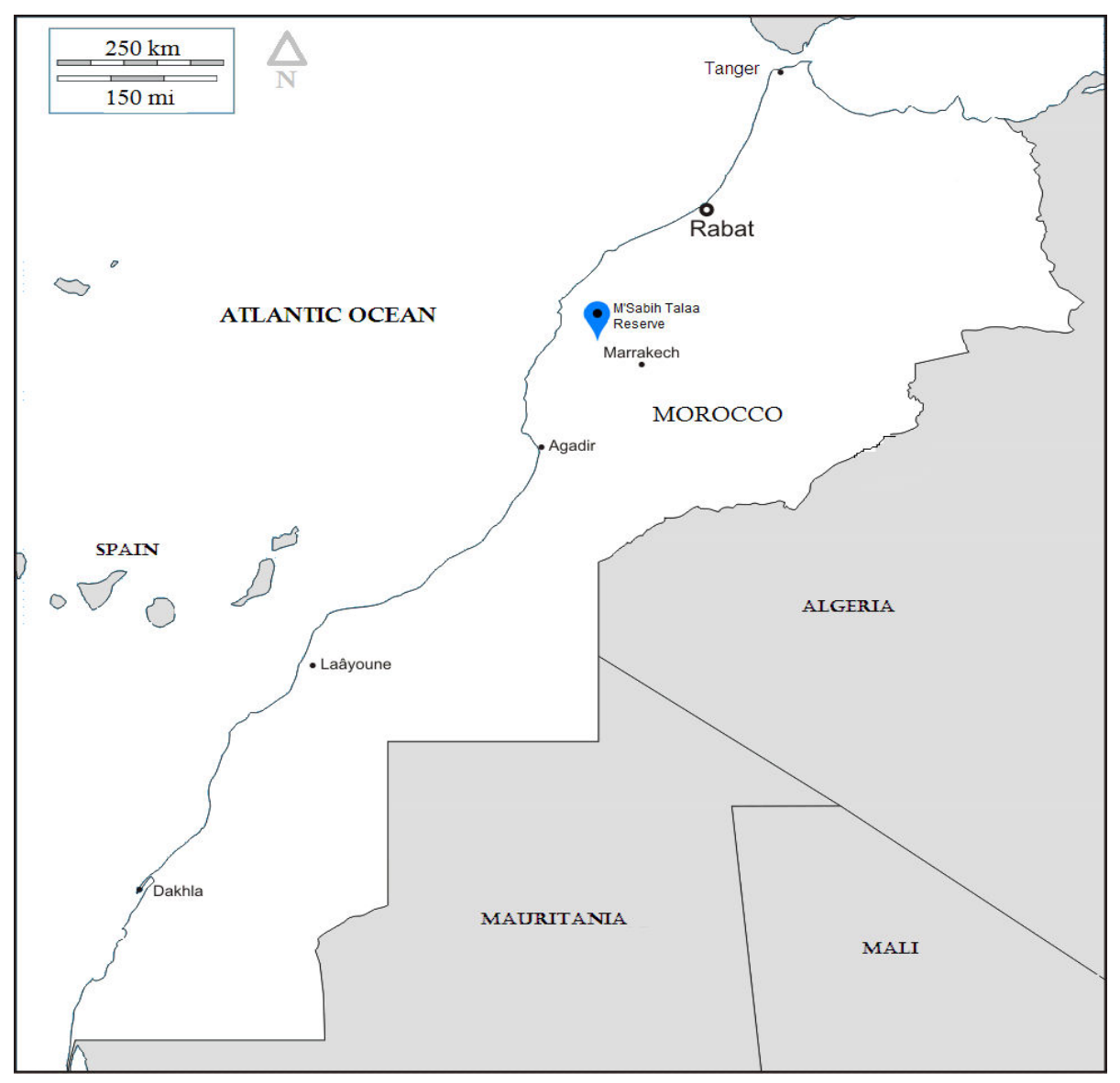

Fig. 1: Map of Morocco showing the location of the M'Sabih Talaa Reserve

data with the particular aim of estimating the population's relative susceptibility to inbreeding depression and catastrophes ("sensitivity testing") and to assess the relative effect of suggested management measures. In particular, we used our data set to infer the consequences of splitting the population into two subpopulations to reduce its susceptibility to environmental stochasticity as was suggested by Cuzin et al. (2007) and Godhino et al. (2012).

\section{Materials and Methods}

Experiments:

Among the main criteria adopted by the IUCN (International Union for the Conservation of Nature) for the classification of threatened species in various categories, the fifth and last criterion "E" is based on the quantitative assessment of the extinction risk. In conservation biology, this assessment is called Population Viability Analysis (PVA) which consists on the examination and analysis of factors placing a species or a local population in danger (Lacy, 1993). The PVA is so a decision-making tool in management and conservation of endangered species. Its use has increased significantly during the last decades and became a credible means for the appreciation of the viability of a species (Soulé, 1987; Boyce, 1992; Beissenger and Westphal, 1998). The models used to predict the viability of a population take into account all the threats which could affect the growth and the sustainability of the small populations, including either demographic 
or environmental stochasticities, the catastrophes, the depression due to the inbreeding and the deterministic pressures as the loss and the destruction of habitats (Reed et al., 2002). The only known PVAs carried out in gazelles are those on the Przewalski's gazelle Procapra przewalskii (Büchner, 1891) in China (Li and Jiang, 2002) and the Goitered gazelle Gazella subguttorosa (Güldenstädt, 1780) in Turkey (Çobanoğlu, 2010). This kind of analyses requires the acquisition of accurate data on various demographic parameters, life history and even genetic traits.

We present the use of the PVA in the remnant population of the Moroccan dorcas gazelle in the MT reserve. We used VORTEX (Lacy, 1993, 2000) to simulate the dynamics of the MT population. This analysis should be considered as a preliminary because of the lack of some demographic and genetic data, particularly actual age-specific mortality rates and inbreeding coefficient.

Few demographic data are available for dorcas gazelle in the wild (Baharav, 1980; Loggers, 1992; Lawes and Nanni, 1993; Marraha, 1996) and data on survivorship are particularly lacking. In the wild, uncertainty about the survival status of animals lost to follow up and imprecision of age assignment are important obstacles to the accurate assessment of survival. Both in the wild and in captivity, the small number of animals typically under observation entails substantial variability in estimates. In order to address this deficiency of data on dorcas gazelle survival in MT, we used a period life table of captive dorcas gazelle based upon age-specific mortality rates observed during the period from January 1, 1998 to December 31, 2003 (Kohler et al., 2006). Such a period life table follows a synthetic cohort of births and assumes that they are subject at each age to the age-specific death rates observed during a particular period (Preston et al., 2001). Although the mortality profile in captivity can be expected to differ from that in the wild, and given that the longevity is about the same (910 years), we can assume that the age-pattern of mortality in dorcas gazelle is similar in both cases with mortality rate rising with age above the juvenile stage. The animals in captivity have the benefits of veterinary care, a lack of predators, and a regular supply of food. On the other hand, captive animals may suffer from higher levels of obesity (Taylor and Poole, 1998; Ward et al., 2003), injuries from exhibits (Leong et al., 2004), and poor adaptation to captivity or to a zoo's climate (Karstad and Sileo, 1971; Gozalo and Montoya, 1991) and from inbreeding that results in higher perinatal mortality (Wielebnowski, 1996). Although, the age-specific mortality rates in captivity can be considered as the minimal levels for small isolated wild populations which are facing predation and diseases and are often subject to inbreeding depressions.

The PVA was carried out using the VORTEX V9.99b software (Lacy 1993) and testing two categories of scenarios (Tables 1, 2).

The basic scenarios were conceived with the aim of estimating the average mortality rate which would have prevailed over a period of 22 years, since the first population size estimate in 1987 (Loggers, 1990, 1992), and that would have resulted in the present 
Table 1: Summary of the various scenarios used in the population viability analysis of Gazella dorcas massaesyla from M' Sabih Talaa reserve, West central of Morocco

\begin{tabular}{|c|c|c|c|c|c|}
\hline \multirow{2}{*}{ Scenario } & & \multirow{2}{*}{ Population size } & \multirow{2}{*}{ Mortality rate } & \multicolumn{2}{|c|}{ Catastrophe's impact } \\
\hline & & & & Survival & Reproduction \\
\hline \multirow{4}{*}{ Basic scenario } & 1 & \multirow{4}{*}{209 individuals } & $100 \%$ the rate in captivity & - & - \\
\hline & 2 & & $150 \%$ the rate in captivity & - & - \\
\hline & 3 & & $200 \%$ the rate in captivity & - & - \\
\hline & 4 & & $250 \%$ the rate in captivity & - & - \\
\hline Single & No Option & \multirow{3}{*}{99 individuals } & $200 \%$ the rate in captivity & $-50 \%$ & $-25 \%$ \\
\hline population & Option 1 & & $250 \%$ the rate in captivity & $-50 \%$ & $-25 \%$ \\
\hline scenario & Option 2 & & $300 \%$ the rate in captivity & $-25 \%$ & $-10 \%$ \\
\hline Splitting & No Option & $\begin{array}{l}\text { Two populations: } \\
66 \text { and } 33 \text { individuals }\end{array}$ & $200 \%$ the rate in captivity & $-50 \%$ & $-25 \%$ \\
\hline
\end{tabular}

Table 2: VORTEX required data for the viability analysis of the population of Gazella dorcas massaesyla in the M' Sabih Talaa reserve, West central Morocco.

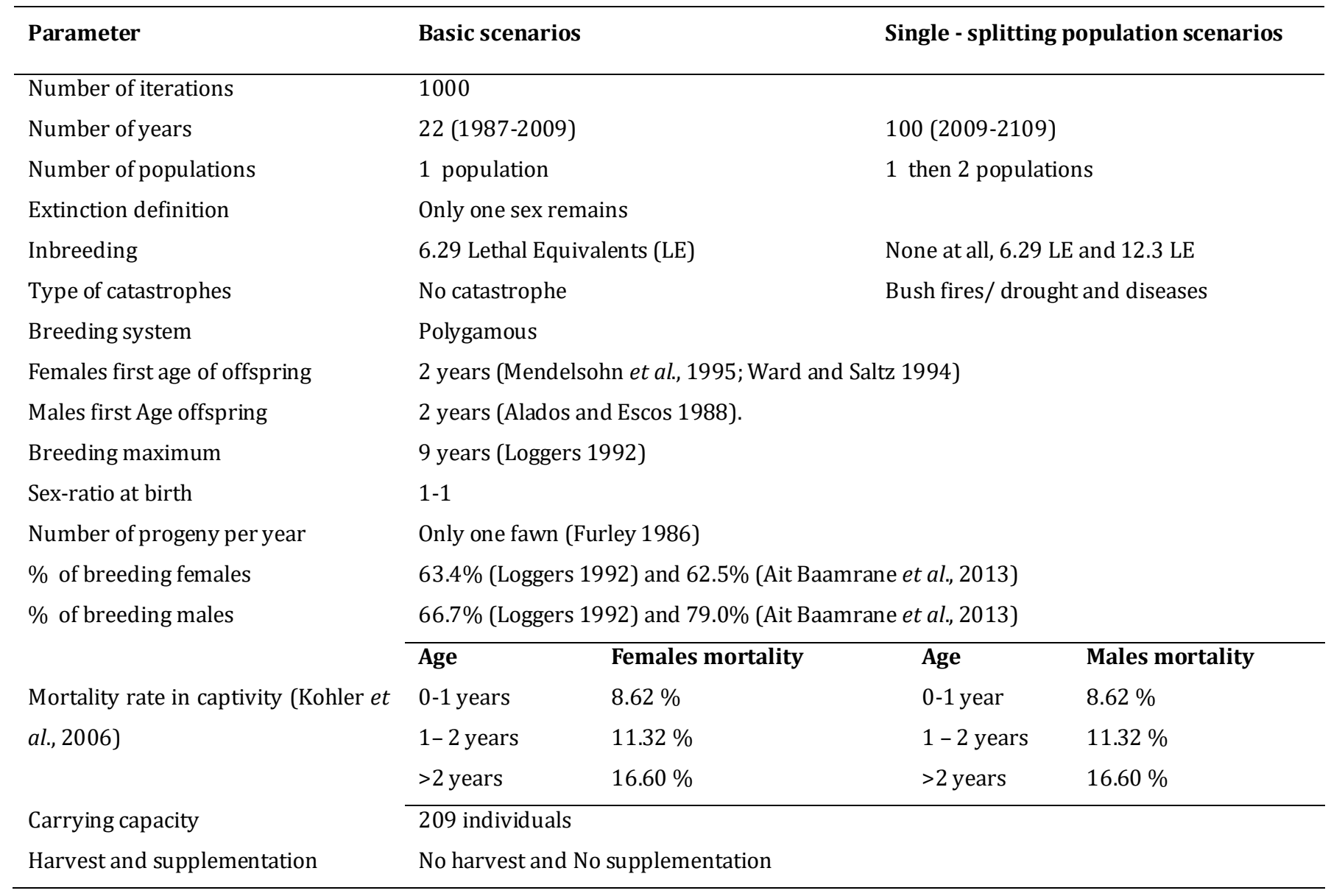


population size obtained by line transect method (Ait Baamrane et al., 2013). In this purpose, we tested four levels $(100,200,250$ and $300 \%)$ of the age-specific mortality rates reported by Kohler et al. (2006) for captive dorcas gazelle. The initial size of the MT dorcas gazelle population in 1987 was 209 individuals according to Loggers (1992).

On the basis of the likely mortality rates of the population during the last two decades (1987 to 2009), different scenarios were tested to predict the likely trend of the population size over a period of the forthcoming 100 years; the initial population size considered in this analysis was that obtained in the last line transect session (April 2009).

The VORTEX software has been shown to produce especially good results in a comparative study of PVA programs (Brooks et al., 2002). In particular, we carried out a sensitivity analysis to infer which parameters are most important to the population's viability. By using a range of possible values for uncertain parameters we can determine what effects these uncertainties are likely to have on the PVA outputs. This approach has been proved to be particularly fruitful in conservation and management (Frankham et al., 2002; Allendorf and Luikart, 2007). We specifically tested the relative effects of inbreeding depression, catastrophes, and splitting the population into two subpopulations. Inbreeding depression was chosen because the MT gazelle population has been isolated with no supplementation for a long time (more than 60 years) resulting in a low genetic variability (Lerp et al., 2011; Godinho et al., 2012) and has drastically declined during the last 15 years and genetic consequences of the long-term low effective population size and the severe bottleneck of the MT dorcas gazelle are likely to be relevant to their viability. Catastrophes such as bush fires (abundant dry grasses in late springsummer) or diseases (contamination risks from neighboring sheep and goat livestock that sometimes illegally invades the reserve), even uncommon, constitute a serious potential risk on a unique population confined to a small area. The effect of establishing two subpopulations with a higher total carrying capacity was assessed because this approach would reduce the gazelle's susceptibility to environmental stochasticity compared to that of a single population.

We applied three different levels of inbreeding depression in terms of diploid lethal equivalents (LE) $(0,6.29$ and $12.3 \mathrm{LE})$ with a lethal-sublethal ratio of 1:1 (Simmons and Crow, 1977; Brooks et al., 2002). The value of 6.29 LEs per diploid individual is the combined mean effect of inbreeding on fecundity and first year survival reported by 0'Grady et al. (2006). The higher value of 12.3 LEs is the average value obtained in a metaanalysis of inbreeding depression across the life-history of 30 species of mammals and birds (O'Grady et al., 2006).

We considered two types of catastrophes in our analyses: an environmental catastrophe (bush fires/drought) and diseases each with a probability of $0 \%, 1 \%$ or $3 \%$. The severity of these catastrophes was defined as follows: while bush fires/droughts cause a decrease in breeding and survival rate to $75 \%$ of the mean, diseases are expected to be more harmful and reduce these two parameters to $50 \%$ of their mean. These arbitrary values are in concordance with those used for other 
terrestrial mammal species (e.g., Lions Panthera leo (Packer et al., 1999) and Red deers Cervus elaphus (Zachos et al., 2009).

Carrying capacity is difficult to estimate, but we consider the highest population estimate (209 ind. in 1987) ever reported (Loggers, 1992) and the value (418 ind.) based on the suggestion that the MT population might be divided into two subpopulations of about 209 animals.

Since the splitting of the MT population has been suggested as a conservation measure (Cuzin et al., 2007), we also simulated possible effects of this scenario. We based our simulations on two subpopulations both with a carrying capacity of 209 individuals and initial population sizes of $2 / 3$ and $1 / 3$ of the last population estimate (April 2009), respectively for the first subpopulation and second subpopulation. For this latter, the animal group to be translocated from the first to another area would have a sex-ratio of 1.64 females per male, the mean value reported in the present study. Establishing several subpopulations from a single endangered population is an appropriate way of minimizing the susceptibility to environmental stochasticity (Frankham et al., 2002; Zachos et al., 2009). To reduce the inbreeding rate, a translocation of an average one adult female per year between the two subpopulations was incorporated into the calculations as a probably sufficient amount of artificial gene flow. The simulations for a single population and two subpopulations were all run with the same demographic data as indicated in Table 2, but based on lower mortality rates of $125 \%$ the values recorded in captivity (Management option 1). This mitigation could, for instance, be achieved by suppressing the depredation by feral dogs and the effect of poaching.

As the impact of catastrophes on population viability revealed to be important, we also ran simulations with less severe consequences of catastrophic events (decrease in reproduction and survival rate to $90 \%$ and $75 \%$ of the mean for bush fires/drought and diseases, respectively), but with the same mortality rate as in management option 1 for the single population and two subpopulations (Management option 2). This management measure could be achieved by veterinary treatment (vaccinations) and effective fire prevention or by additional management measures aiming at enhancing the fitness of surviving animals. We compared the prospects of the PVAs without management, with management option 1 and with management option 2 for a single population and two subpopulations and between these two cases.

\section{Statistical analysis:}

All the statistical tests were carried out using SPSS program (Version 10.0). The mean values are given with their standard deviation. The Wilcoxon test was used to test the effect of the different variables on the viability parameters. The differences are considered statistically significant at a threshold lower than $5 \%$.

\section{Results}

The basic scenarios 1, 2, 3, 4 and 5 have aim to estimate the mean mortality rate having prevailed under natural conditions during the last two decades (from 1987 to 2009). The results obtained for these five scenarios are given in the Table 3 . 
Table 3: Results of the simulation by VORTEX of the population viability analysis of the M'Sabih Talaa reserve based Moroccan dorcas gazelle population (Basic scenarios)

\begin{tabular}{llllll}
\hline & & \multicolumn{3}{c}{ Mortality Rate } \\
& $\mathbf{1 0 0 \%}$ & $\mathbf{1 5 0 \%}$ & $\mathbf{2 0 0 \%}$ & $\mathbf{2 5 0 \%}$ & $\mathbf{3 0 0 \%}$ \\
\hline PE & 0.00 & 0.00 & 0.00 & 0.015 & 0.331 \\
TFE & - & - & - & 20.07 & 18.58 \\
GR & 0.249 & 0.168 & 0.081 & -0.024 & -0.140 \\
NE & 204 & 196 & 162 & 96 & 24 \\
& 96.53 & 96.36 & 95.75 & 92.03 & 82.13
\end{tabular}

PE: Probability of extinction, TFE: Mean time to the first extinction, GR: Growth rate, N: Non-extinct population size, HE: Heterozygosity expected.

The basic scenarios 1, 2 and 3 showed that the MT gazelle population would have a population size of 204, 196 and 162 individuals, respectively and corresponding mean growth rates (GR) of $0.249 \pm 0.185$; $0.169 \pm 0.197$, respectively and $0.081 \pm 0.208$ per year; the extinction probability (PE) remains null.

According to the basic scenario 4, the obtained population size in 2009 was 96 individuals with a negative mean GR of $0.024 \pm 0.236 /$ year. Under this scenario the risk of extinction is $1.5 \pm 0.004 \%$ at an average time of 20.07 years to the first extinction. With the basic scenario 5, an average population size of only 24 individuals would be reached in 2009. In that case, GR would be $14 \pm 0.274 \%$ per year with an extinction probability of $33.1 \pm 0.015 \%$ and an average time of 18.58 years to the first extinction. The genetic variety which is indicated by the heterozygosity rate (Lacy, 2000; Miller and Lacy, 2005) would decrease with increasing mortality rate (e.g., from $96.53 \%$ to $82.13 \%$, respectively under the basic scenarios 1 and 5).
These results showed that the mortality rate which would have prevailed for 22 years (1987-2009) and which would have given the current population size in the MT reserve would be similar to that of the basic scenario 4 which is $250 \%$ the mortality rate reported in captivity. This rate would result in a population size of 96 individuals in 2009, which is quite similar to the average estimate obtained in 2009 (102 ind.; range: 95-111).

The comparison between the simulations with and without management options expectedly yielded generally better prospects for the first case (Table 4). The mean probability of extinction in 9 runs (each possible combination of the three levels of inbreeding depression and catastrophe probability; carrying capacity was 209 for a single population) was $93.83 \%$ without and $72.18 \%$ and $60.92 \%$, respectively with management options 1 and 2 (significant differences, Wilcoxon test: no option vs. option 1 and no option vs. option $2, \mathrm{Z}=-2.666$, $\mathrm{p}=0.008$ in both cases and option 1 vs. option $2: \mathrm{Z}=-2.549, \mathrm{p}=0.011$ ). The respective values for final expected heterozygosity (relative to 
Table 4: Effect of single population scenarios on extinction probability (PE), growth rate (GR), expected heterozygosity (HE) and Non-extinct population size (N) of the M'Sabih Talaa reserve based dorcas gazelle population.

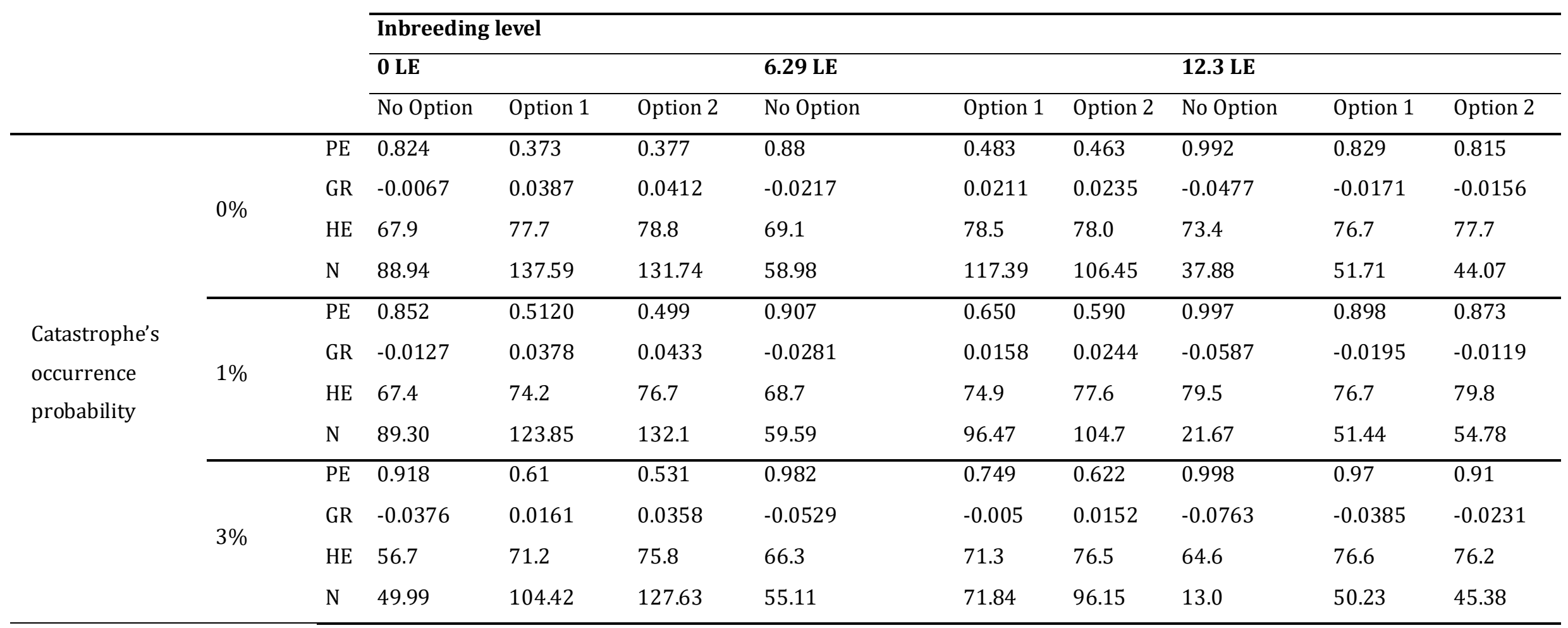


generation 1 whose heterozygosity is defined as $100 \%$ ) were $62.0 \%$ without vs. $74.1 \%$ and $76.7 \%$, respectively with management options 1 and 2 (significant difference, Wilcoxon test: no option vs. option $1: \mathrm{Z}=-2.547, \mathrm{p}=0.011$, no option vs. option 2: $\mathrm{Z}=-2.666 \mathrm{p}=0.008$, and option 1 vs. option $2: \mathrm{Z}=-2.310, \mathrm{p}=0.021$ ), and the corresponding final sizes of non-extinct populations averaged 47.52 vs. 85.6 and 90.05 individuals (significant difference, Wilcoxon test: no option vs. option 1: Z= 2.666, $\mathrm{p}=0.008$ and no option vs. option $2: \mathrm{Z}=-$ $2.668 \mathrm{p}=0.008$, but not for option 1 vs. option $2: \mathrm{Z}=-0.770, \mathrm{p}>0.05$ ).

Both inbreeding depression and catastrophes had a large impact on the population's viability parameters. Prospects were especially more pessimistic with a combination of a high inbreeding depression and a high probability for the occurrence of catastrophes. However, the buffering effect of the management option 1 and particularly option 2 was considerable. Apart from the significantly different means given above it is of particular interest that these measures were able to largely compensate for the consequences of inbreeding and that negative stochastic growth rates were only found for worst-case scenarios (with inbreeding depression of 12.3 LE and any catastrophe level) whereas with no management measures were never positive.

Splitting of the MT population into two subpopulations further mitigated the negative consequences of inbreeding depression and catastrophes (Table 5). Except the cases without management measures and two subpopulations with management option 1 vs. Single population with management option 2 , mean probability of extinction and relative expected heterozygosity - were statistically significantly more favorable under the twosubpopulations-scenarios than they were when assuming only one population. However, apart from the comparisons of two subpopulations with management option 2 vs. Single population with management option 1 and subpopulations with management option 1 vs. Single population with management option 2, respectively for the corresponding stochastic growth rates and final population sizes, the mean values are statistically better in all other cases under the splitting population-scenarios than they were only under single population scenarios.

Comparisons among the different splitting population scenarios, attested that mitigation of the effect of catastrophic events (management option 1 and 2) revealed to have a high potential to increase population viability. With management option 2 the two subpopulations performed much better than under management option 1 . All the differences were statistically significant for all four parameters, and this also yields for the worst-case scenario: extinction probability was reduced by $15 \%$, population decline was three times lower and final population size was $2 / 3$ of the initial one instead of a total extinction without any management option.

\section{Discussion}

Small populations are subject to whims of breeding and natural catastrophes, and are vulnerable to extinctions due to demographic and environmental stochasticities (Soulé 1987) and genetic constraints (Bijlsma et al., 2000). Demographic stochasticity influences how many offspring are produced, and to what extent adults can bred successfully, while the environmental stochasticity determines the survival and the fertility of the population 
Table 5: Effect of splitting population scenarios on extinction probability (PE), growth rate (GR), expected heterozygosity (HE) and Non-extinct population size (N) of the M'Sabih Talaa reserve based dorcas gazelle population.

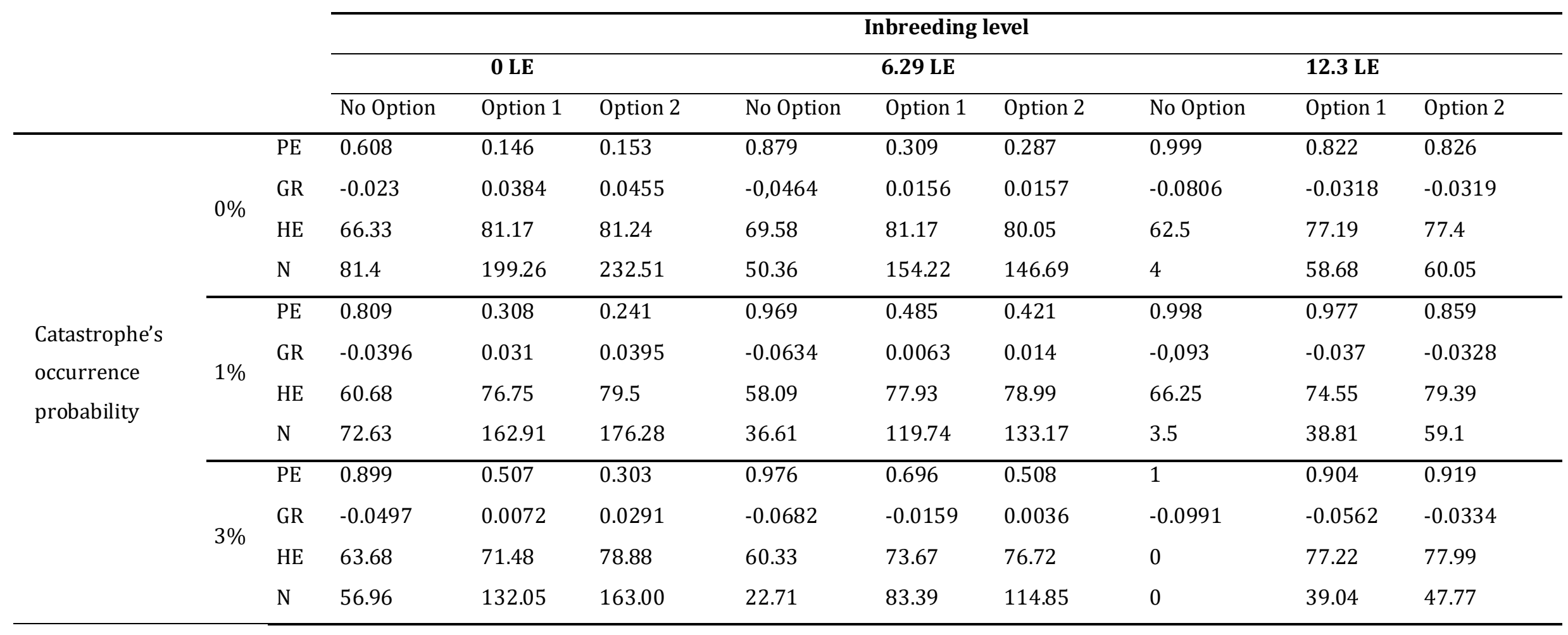


by its effects on the food supply. Genetic stochasticity influences the change in frequency of desirable or harmful alleles between generations (Stephens and Sutherland, 1999; Bhatnagar et al., 2007). All these stochastic, and the aforementioned deterministic factors can precipitate the extinction of small populations such as the MT dorcas gazelle. Thus, although, predictable factors such as poaching and predation by feral dogs may bring about population declines, the final extinction is often a result of smallness of a population that is unable to recover from short-term disturbances.

It is a general problem of PVAs that for some of the required input parameters no actual field data are known. Demographic and life-history data for MT dorcas gazelle used in the present work, such as total population size and age-specific mortality rates, were derived respectively from actual field studies and from captive populations of the same species.

Survival was assumed to have a similar pattern as in captivity and was constant and did not change throughout the year. This could be partly true if we consider that in the MT reserve water is supplied during the dry periods, particularly in summer and even food is offered during seasons of low productivity. However, it would be better, if a representative sample of gazelles could be captured, individually marked and surveyed by MarkResight method during a relatively long period, so a complete actual survival rate would be calculated.

As for fecundity, similarly, if a representative number of female gazelles were marked and monitored using the MarkResight method for sufficient periods, detection of calves of females of different ages would allow to determine specifically the actual fecundity rates.

For carrying capacity, this was considered just as the highest total population size reported for the MT dorcas gazelle (209 ind. or a density of 0.105 ind./ha). As was indicated before, the actual carrying capacity is difficult to obtain, but in the case of dorcas gazelle in MT, this could be higher given that the density can be more important in less productive environment such as the Ténéré Reserve with a population density of 0.16 ind./ha (which would correspond to a potential carrying capacity of at least 318 individuals for MT). However, in any further assessment of the actual carrying capacity, one should take into account either the availability of spaces (home range size) or the food requirements in relation with the habitat suitability and productivity.

The inbreeding (number of diploid lethal equivalents per individual) was tested for three different values including the total absence of inbreeding and the median and highest LE known among mammals. The actual inbreeding level has not been determined yet for the MT population, but the present small population size along with the low genetic diversity (Cassinello, 2005), could be associated with a relatively high inbreeding coefficient, but probably not higher than the mean value $(F=0.051)$ reported for captive dorcas gazelles in the EEZA (Spain) (Cassinello, 2005). Indeed, genetic variability of the MT population was found to be lower than other (in Morocco and Spain) semicaptive gazelle populations (heterozygosity: $33.47 \%$ vs. $66.0 \%$ and 2.75 alleles/loci vs $3-$ 4.25 alleles /loci) (Cassinello, 2005). 
The output of our PVA analyses confirm that given the presumed demographic data and history of the MT reserve dorcas gazelle, inbreeding depression has an important impact on extinction risk and other viability parameters. The difference between 12.3 and 6.29 lethal equivalents was clearly more distinct than the one between 6.29 and 0 LEs. Unfortunately, 12.3 LEs are probably much more realistic than 6.29 as the latter value was calculated taking into account only life-time breeding success, whereas the higher number of 12.3 was estimated across the life-history and thus encompasses more fitness parameters than breeding success alone (O'Grady et al., 2006). Our analysis generated pessimistic expectations for the MT dorcas gazelle population with high probabilities of extinction, but we could also show that management measures, in particular the establishment of two subpopulations with artificial gene flow among them and mitigation of the effect of annual all class mortality, are able of significantly reducing the risk of extinction and the critical decay of genetic variability as indicated by the final expected heterozygosity levels.

One of the objectives in the management of endangered populations is to retain $90 \%$ of their genetic diversity for 100 years (Soulé, 1987; Frankham et al., 2002). Given the very low initial genetic diversity of the MT reserve dorcas gazelle (0.3347) and taking into account that heterozygosity is correlated to fitness in many cases (Reed and Frankham, 2003), management actions should explicitly aim at creating conditions under which as little heterozygosity as possible will be lost in the future.

Artificially increasing genetic diversity through introduction of non-related dorcas gazelle (as was done with the Florida panther Puma concolor coryi (Pimm et al., 2006)) should be absolutely avoided as this would mean polluting the genetic integrity of this unique gazelle population. Indeed, with its clear genetic differentiation from other dorcas gazelle (Godinho et al., 2012), the MT reserve population clearly meets the criteria of being treated as a separate management unit (Moritz and Cicero, 2004). Our sensitivity analyses have shown that there are alternatives to the introduction of animals of which the establishment of two subpopulations (with artificial gene flow) and the mitigation of the impact of mortality rate seem to be particularly promising. By implementing these measures, loss of heterozygosity and probability of extinction can be further reduced. If actual inbreeding depression reveals to be obviously decisive factor, then the final options could be to artificially increase the effective population size by equalizing family sizes, removing longterm reproductive males and exchanging more individuals between subpopulations once established (splitting population scenario).

Variation of catastrophic impacts showed that this parameter was crucial as was the probability of the occurrence of catastrophic events. This is the strength of sensitivity analyses - applying a range of values for uncertain input parameters and having an idea of the relative effect of different parameters on population viability (Miller and Lacy, 2005).

The most important results of our simulations are the relative increase in viability with management measures (suppression of poaching and predation by feral dogs), and by duplicating the original population. These management measures led 
to significantly better prospects in our simulations and were shown to be valuable tools for the future management and conservation of the MT reserve population. As these gazelles are demographically nonperformant, it might be assumed that the establishment of a second population in suitable similar habitat will show better demographic characteristics, in particular a higher fecundity rate and a higher proportion of juveniles.

Before splitting the population into two subpopulations, an important step to be undertaken simultaneously is to clearly identify all areas where Moroccan dorcas gazelle were known to occur in the past on the Plateaux's Atlantic coast. The potential threats to gazelles or their habitat in these sites will need to be understood, and their suitability for restoration assessed based on the habitat quality, human pressures, and potential for connectivity (even artificial) with the original population. After identification of the most suitable site, habitat restoration, addressing potential threats to the gazelles, and enlisting local support for gazelle reintroduction will need to be undertaken. Constant monitoring and research will assist in assessing the suitability of the selected site for reintroduction.

The MT reserve dorcas gazelles are the last remaining genetic legacy from the formerly widespread Moroccan dorcas gazelle population on the Plateaux's Atlantic coasts. A large body of data from a long term population survey is needed to improve the PVA and to provide local authorities with further information to realize the recommendations of the HCEFLCD (Haut Commissariat aux Eaux \& Forêts \& à la Lutte
Contre la Désertification) report (Cuzin et al., 2007) and to estimate the costs involved in this. Further, our study highlights the positive effects of population splitting, a conservation measure that is also being carried out in other species (e.g., the Asiatic lion Panthera leo persica (Johnsingh et al., 2007), the Red deer Cervus elaphus (Zachos et al., 2009) and that may turn out to be of great value in conservation biology in general.

\section{References}

Ait Baamrane MA, Znari M, Loggers CO, El Mercht S and Naimi M. (2013) Demographic decline of the last surviving Moroccan dorcas gazelles Gazella dorcas massaesyla in M'Sabih Talaa Reserve, Morocco. Fauna Flora Intern Oryx 47:578-583.

Ait Baamrane MA, Znari M, Loggers CO, Naimi M and El Mercht S. (2009) Conservation and management of an isolated remnant population of Moroccan Dorcas Gazelles North West of the Atlas Mountains. Second DIVERSITAS Open Science Conference (OSC2), Cape Town, South Africa 13-16 October 2009.

Alados CL. (1987) A cladistic approach to the taxonomy of the dorcas gazelle. Israel J Zool. 34:33-49.

Alados CL and Escos J. (1988) Parturition dates and mother-kid behavior in Spanish ibex (Capra pyrenaica) in Spain. J Mammalogy 69:172-175.

Allendorf FW and Luikart G. (2007) Conservation and the genetics of populations. Blackwells, Oxford, pp. 642

Baharav D. (1980) Habitat utilization of the dorcas gazelle in a desert saline area. J Arid Environ. 3: 161-167.

Beissenger SR and Westphal MI. (1998) On the use of demographic models of population viability in endangered species management. J Wildlife Managem. 62:821-841.

Bhatnagar YV, Seth CM, Takpa J, Ul-haq S, Namgail T, Bagchi S and Mishra C. (2007) A strategy for conservation of the Tibetan gazelle Procapra picticaudata in Ladakh. Conserv Soc. 5:262-276.

Bijlsma R, Bundgaard J and Boerema AC. (2000) Does inbreeding affect the extinction risk of small populations?: predictions from Drosophila. J Evolutionary Biol. 13: 502-514.

Boyce MS. (1992) Population viability analysis. Ann Rev Ecol Sys. 23:481-506. 
Brooks TM, Mittermeier RA, Mittermeier CG, Da Fonseca GAB, Rylands AB, Konstant WR, Flick P, Pilgrim J, Oldfield S, Magin G and Hilton-Taylor C. (2002) Habitat loss and extinction in the hotspots of biodiversity. Conser Biol. 16: 909-923.

Cassinello J. (2005) Inbreeding depression on reproductive performance and survival in captive gazelles of great conservation value. Biol Conser. 122: 453-464.

Çobanoğlu AE. (2010) Identification of demographic structure and population viability analysis of Gazella subgutturosa in Şanlıurfa. Ms Thesis, Middle East Technical University, Ankara, Turkey.

Cuzin F. (1996) Réparation actuelle et statut des grands mammifères sauvages $\mathrm{du}$ Maroc (Primates, Carnivores, Artiodactyles). Mammalia 60: 101-124.

Cuzin F. (2003) Les grands Mammifères du Maroc méridional (Haut Atlas, Anti Atlas, Sahara): Distribution, écologie et conservation. Thèse de Doctorat, Ecole Pratique des Hautes Etudes (EPHE), Montpellier II, Montpellier: p. 348.

Cuzin F, Sehhar EA and Watcher T. (2007) Etude pour l'élaboration de lignes directrices et d'un plan d'action stratégique pour la conservation des ongulés au Maroc. Haut Commissariat aux Eaux et Forêts et à la Lutte contre la Désertification, Projet de Gestion des Aires Protégées (PGAP) et Banque Mondiale, Global Environment Facility 2 volumes.

Frankham R, Ballou JD and Briscoe DA. (2002) Introduction to conservation genetics. Cambridge University Press, USA.

Furley CW. (1986) Reproductive parameters of African gazelles: gestation, first fertile matings, first parturition and twinning. African J Ecol. 24:121-128.

Gentry AW. (1971) Les Gazelles. In: The mammals of Africa: an identification manual, (eds.) Meester J and Setzer HW, Smithsonian Institution Press, Washington.

Godinho R, Abaigar T, Lopes S, Essalhi A, Ouragh L, Cano $\mathrm{M}$ and Ferrand N. (2012) Conservation genetics of the endangered dorcas gazelle (Gazella dorcas spp.) in northwestern Africa. Conser Gen. 13: 1003-1015.

Gozalo A and Montoya E. (1991) Mortality causes of the moustached tamarin (Saguinus mystax) in captivity. J Med Primatol. 21:35-38.

Housawi FMT, Abu Elzein EME, Mohamed GE, Gameel AA, Al-Afaleq AL, Hegazi A and Al-Bishr B. (2004) Emergence of peste des petits ruminants in sheep and goats in eastern Saudi Arabia. Revue Élev Méd Vét Pays Trop. 57: 31-34.
IUCN S. (2008) Gazella dorcas. IUCN Red List of Threatened Species v. 2012.2. http://www.Iucnred list. Org.

Johnsingh AJT, Goyal SP and Qureshi Q. (2007) Preparations for the reintroduction of Asiatic lion Panthera leo persica into Kuno Wildlife Sanctuary, Madhya Pradesh, India. Oryx 41: 93-96.

Karstad L and Sileo L. (1971) Causes of death in captive wild waterfowl in the Kortright Waterfowl Park, 1967-1970. J Wildl Dis. 7: 236-241.

Kohler I, Prestion SH and Lakey LB. (2006) Comparative mortality levels among selected species of captive animals. Demog Res. 15: 413-434.

Lacy RC. (1993) VORTEX, a computer simulation for use in population viability analysis. Wildl Res. 20: 45-65.

Lacy RC. (2000) Structure of the VORTEX simulation model for population viability analysis. Ecol Bull. 48: 191-203.

Lawes MJ and Nanni RF. (1993) The density, habitat use and social organization of dorcas gazelles (Gazella dorcas) in Makhtesh Ramon, Nagev Desert, Israel. J Arid Environ. 24:177-196.

Leong KM, Terrell SP and Savage A. (2004) Causes of mortality in captive cotton-top tamarins (Saguinus oedipus). Zoo Biol. 23: 127-137.

Lerp H, Wronski T, Pfenninger M and Plath M. (2011) A phylogeographic framework for the conservation of Saharan and Arabian dorcas gazelles (Artiodactyla: Bovidae). Organisms Diver Evol. 11: 317-329.

Li D and Jiang Z. (2002) Population viability analysis of the Przewalski's gazelle. Russian J Ecol. 33:115-120.

Loggers CO. (1990) Food habits and population characteristics of dorcas gazelles, and distributions and statuses of wild ungulates in Morocco. Graduate Student Theses, Dissertations, and Professional Papers. 7373. https://scholarworks.umt.edu/etd/ 7373.

Loggers CO. (1992) Population characteristics of dorcas gazelles in Morocco. Afr J Ecol. 30: 301-308.

Loggers CO, Thévenot M and Aulagnier S. (1992) Status and distribution of Moroccan wild ungulates. Biol Conser. 59: 9-18.

Marraha M. (1996) Utilisation du line transect dans l'estimation de la densité et des caractéristiques de la population de gazelle dorcas (Gazella dorcas L.) dans la réserve de M'Sabih Talaa. Ann Recher Forest Rabat Morocco 29: 40-49.

Mendelsohn H, Yom-Tov Y and Groves C. (1995) Gazella gazella. Mammalian Species 490: 1-7. 
Miller PS and Lacy RC. (2005) VORTEX: A Stochastic simulation of the extinction process. Version 9.50 User's Manual. Conservation Breeding Specialist Group (SSC/IUCN).

Moritz C and Cicero C. (2004) DNA Barcoding: Promise and Pitfalls. PLoS Biol. 2: e354.

O'Grady JJ, Brook BW, Reed DH, Ballou JD, Tonkyn DW and Frankham R. (2006) Realistic levels of inbreeding depression strongly affect extinction risk in wild populations. Biol Conser. 133: 42-51.

Packer C, Altizer S, Appel M, Brown E, Martenson J, O'Brien SJ, Roelke-Parker M, Hofmann-Lehmann R and Lutz H. (1999) Viruses of the Serengeti: patterns of infection and mortality in African lions. J Anim Ecol. 68:1161-1178.

Panouse JB. (1957) Les mammifères du Maroc. Travaux de l'Institut Scientifique Chérifien, Série Zoologique 5:1-206.

Pimm SL, Dollar L and Bass OL. (2006) The genetic rescue of the Florida panther. Anim Conser. 9: 115-122.

Preston SH, Heuveline P and Guillot M. (2001) Demography. Measuring and Modeling Population Processes. Oxford Blackwell.

Reed DH and Frankham R. (2003) Correlation between fitness and genetic diversity. Conser Biol. 17: 230-237.

Reed JM, Mills LS, Dunning JB, Menges ES, Kelvey KSM, Frye CR, Beissinger SR, Anstett MC and Miller P. (2002) Emerging issues in population viability analysis. Conser Biol. 16: 7-19.
Simmons MJ and Crow JF. (1977) Mutations affecting fitness in Drosophila populations. Annu Rev Gen. 11: 49-78.

Soulé ME. (1987) Viable populations for conservation. Cambridge University Press, Cambridge, UK.

Stephens PA and Sutherland WJ. (1999) Consequences of the Allee effect for behavior, ecology, and conservation. TREE 14: 401-405.

Taylor VJ and Poole TB. (1998) Captive breeding and infant mortality in Asian elephants: A comparison between twenty western zoos and three eastern elephant centers. Zoo Biol. 17: 311-332.

Ward D and Saltz D. (1994) Forging at different spatial scales: Dorcas gazelles foraging for lilies in the Negev Desert. Ecology 75:48-58.

Ward MP, Ramer JC, Proudfoot J, Garner MM, Juan-Sallès $\mathrm{C}$ and Wu CC. (2003) Outbreak of salmonellosis in a zoologic collection of Lorikeets and Lories (Trichoglossus, Lorius, and Eos spp.). Avian Dis. 47: 493-498.

Wielebnowski N. (1996) Reassessing the relationship between juvenile mortality and genetic monomerphism in captive cheetahs. Zoo Biol. 15: 353-369.

Zachos FE, Hajji GM, Hmwe SS, Hartl GB, Lorenzini R and Mattioli S. (2009) Population viability analysis and genetic diversity of the endangered red deer Cervus elaphus population from Mesola, Italy. Wildl Biol. 15: 175-186. 